PROCEEDINGS OF THE

AMERICAN MATHEMATICAL SOCIETY

Volume 128, Number 4, Pages 1147-1155

S 0002-9939(99)05084-

Article electronically published on August 5, 1999

\title{
MULTIDIMENSIONAL ANALOGUES OF BOHR'S THEOREM ON POWER SERIES
}

\author{
LEV AIZENBERG
}

(Communicated by Steven R. Bell)

\begin{abstract}
Generalizing the classical result of Bohr, we show that if an $n$ variable power series converges in $n$-circular bounded complete domain $D$ and its sum has modulus less than 1 , then the sum of the maximum of the modulii of the terms is less than 1 in the homothetic domain $r \cdot D$, where $r=1-\sqrt[n]{2 / 3}$. This constant is near to the best one for the domain $D=\left\{z:\left|z_{1}\right|+\ldots+\left|z_{n}\right|\right.$ $<1\}$.
\end{abstract}

\section{Preliminaries}

The following formulation of the Bohr's result [5] (due to the work of M. Riesz, I. Schur and F. Wiener) is known.

Theorem 1. If a power series

$$
\sum_{k=0}^{\infty} c_{k} z^{k}
$$

converges in the unit disk and its sum has modulus less than 1 , then

$$
\sum_{k=0}^{\infty}\left|c_{k} z^{k}\right|<1
$$

in the disk $\{z:|z|<1 / 3\}$. Moreover, the constant $1 / 3$ cannot be improved.

Recently H.P. Boas and D. Khavinson obtained some multidimensional generalizations of this result ([4], see also for more references there).

Denote by $K_{n}$ the largest number such that if the series

$$
\sum_{\alpha} c_{\alpha} z^{\alpha}
$$

converges in the unit polydisk $U_{1}=\left\{z:\left|z_{j}\right|<1, j=1, \ldots, n\right\}$ and the estimate

$$
\left|\sum_{\alpha} c_{\alpha} z^{\alpha}\right|<1
$$

is valid there, then

$$
\sum_{\alpha}\left|c_{\alpha} z^{\alpha}\right|<1
$$

Received by the editors April 28, 1998 and, in revised form, June 8, 1998.

1991 Mathematics Subject Classification. Primary 32A05.

This work was supported by the BSF, grant No 94-00113.

(C)2000 American Mathematical Society 
holds in $K_{n} \cdot U_{1}$; here $\alpha=\left(\alpha_{1}, \ldots, \alpha_{n}\right)$, all $\alpha_{j}$ are non-negative integers, $z=$ $\left(z_{1}, \ldots, z_{n}\right), z^{\alpha}=z_{1}^{\alpha_{1}}, \ldots, z_{n}^{\alpha_{n}}$.

Theorem 2 (Boas, Khavinson). It is true for $n>1$ that

$$
\frac{1}{3 \sqrt{n}}<K_{n}<\frac{2 \sqrt{\log n}}{\sqrt{n}} .
$$

Theorem 3 (Boas, Khavinson). Let the series (3) converge in a complete n-circular domain (Reinhardt domain) $D$ and (4) holds in D. Then (5) is true in the homothetic domain $K_{n} \cdot D$.

Notice that Remark 1 from [4], in fact, contains a result stronger than the left part of the inequality (6), namely

$$
K_{n}> \begin{cases}\frac{2}{5 \sqrt{n}} & \text { for } n>1, \\ \frac{1}{2 \sqrt{n}} & \text { for large enough } n .\end{cases}
$$

\section{MAIN RESUlts}

We consider some other multidimensional variations of Bohr's problem. Denote by $B_{n}(D)$ the largest number $r$ such that if the series (3) converges in a complete $n$-circular bounded domain $D$ and (4) holds in it, then

$$
\sum_{\alpha} \sup _{D_{r}}\left|c_{\alpha} z^{\alpha}\right|<1,
$$

where $D_{r}=r \cdot D$ is the homothetic transformation of $D$. If $D=U_{1}$, then $B_{n}(D)=$ $K_{n}$. We point out that our consideration is also a natural generalization of Bohr's theorem (Theorem 1), because it was shown in [2] that any power series (3), converging in $D$, converges also in the sense of the left part of (7) for all $r, 0<r<1$.

Theorem 4. The inequality

$$
1-\sqrt[n]{\frac{2}{3}}<B_{n}(D)
$$

is true for any complete, bounded $n$-circular domain $D$.

This estimate can be improved for concrete domains.

Theorem 5. For the unit ball $D^{1}=\left\{z:\left|z_{1}\right|^{2}+\ldots+\left|z_{n}\right|^{2}<1\right\}$ the following estimate is true:

$$
B_{n}\left(D^{1}\right)> \begin{cases}\frac{2}{5 n} & \text { for } n>1, \\ \frac{1}{2 n} & \text { for large enough } n .\end{cases}
$$

Theorem 6. For the unit hypercone $D^{\circ}=\left\{z:\left|z_{1}\right|+\ldots+\left|z_{n}\right|<1\right\}$ the following inequality holds:

$$
B_{n}\left(D^{\circ}\right)<\frac{0,446663}{n} .
$$

Corollary.

$$
1-\sqrt[n]{\frac{2}{3}}<B_{n}\left(D^{\circ}\right)<\frac{0.446663}{n}
$$


Remark 1 . The asymptotic equality

$$
1-\sqrt[n]{\frac{2}{3}}=\frac{\log 3 / 2}{n}+O\left(\frac{1}{n^{2}}\right)
$$

is true, where $\log 3 / 2 \approx 0.405465$. Denoting by $B_{-}$the left part of (10) and by $B_{+}$ the right one, we get

$$
1<\lim _{n \rightarrow \infty} \sup \frac{B_{+}}{B_{-}}<1,1016 .
$$

We now turn our attention to a related problem. Denote by $L_{n}\left(D^{\circ}\right)$ the biggest number $r$ such that if the series (3) converges in the hypercone $D^{\circ}$ and (4) holds in it, then

$$
\sum_{\alpha}\left\|c_{\alpha} z^{\alpha}\right\|_{L^{1}\left(\partial D_{r}^{\circ}\right)}<1
$$

where the $L^{1}$-norm is considered with respect to the measure $\mu_{r}$, . The measure $\mu_{r}$ is the image of the measure

$$
d \mu=\frac{(n-1) !}{(2 \pi i)^{n}} d\left|z_{1}\right| \wedge \cdots \wedge d\left|z_{n-1}\right| \wedge \frac{d z_{1}}{z_{1}} \wedge \cdots \wedge \frac{d z_{n}}{z_{n}}
$$

by the homothetic transformation $z \rightarrow r z$. Usually the measure $d \mu$ is used for calculating the Szëgo kernel for $D^{\circ}$ (see [3]); notice that $\mu\left(\partial D^{\circ}\right)=1$. For $n=1$ this problem coincides with Bohr's problem. The analogous problem for the polydisk $U_{1}$ (with $L^{1}$-norm on its Shilov boundary with respect to usual Lebesgue measure on it) is equivalent to the problem considered in Theorem 2.

Theorem 7. For the hypercone $D^{\circ}$ the following estimates are true:

$$
\frac{1}{3 e^{1 / 3}}<L_{n}\left(D^{\circ}\right) \leq 1 / 3
$$

Surprisingly, the estimates in (12) do not depend on $n$. This is different from the results of Theorems $2-6$.

We consider now a variation of the multidimensional Bohr problem, dealing with the expansions into a series of homogeneous polynomials, which is also a natural generalization of the power series expansion.

Let $Q$ be a complete circular domain (Cartan's domain) centered at $0 \in Q$. Then any function $f(z)$, holomorphic in $Q$, can be expanded into the series

$$
f(z)=\sum_{k=0}^{\infty} P_{k}(z)
$$

where $P_{k}(z)$ is a homogeneous polynomial of degree $k$ for every $k \in \mathbf{N}$.

Theorem 8. If the series (13) converges in the domain $Q$ and the estimate $|f(z)|<$ 1 holds in it, then

$$
\sum_{k=0}^{\infty}\left|P_{k}(z)\right|<1
$$

in the homothetic domain $\frac{1}{3} Q$. Moreover, if $Q$ is convex, then $1 / 3$ is the best possible constant.

Next we consider for the hypercone $D^{\circ}$ the same problem, which was considered in Theorems 2-3 for $U_{1}$. Denote by $K_{n}\left(D^{\circ}\right)$ the largest number such that if series (3) converges in $D^{\circ}$ and estimate (4) is valid there, then (5) holds in $K_{n}\left(D^{\circ}\right) \cdot D^{\circ}$. 
Theorem 9. For the hypercone $D^{\circ}$ the following estimates are true:

$$
\frac{1}{3 e^{1 / 3}}<K_{n}\left(D^{\circ}\right) \leq 1 / 3
$$

Moreover, if $z \notin 1 / 3 \cdot D^{\circ}$, then there exists a series of the form (3) such that it converges in $D^{\circ}$ and the estimate (4) is valid there, but (5) fails at the point $z$.

\section{Proofs}

Proof of Theorem 4. The following generalization of Cauchy inequalities was considered in [2]: if (4) holds in $D$, then

$$
\left|c_{\alpha}\right| \leq \frac{1}{d_{\alpha}(D)}
$$

where $d_{\alpha}(D)=\max _{\bar{D}}\left|z^{\alpha}\right|$. Using Wiener's method, it is easy to strengthen the estimates (15):

$$
\left|c_{\alpha}\right| \leq\left(1-\left|c_{0}\right|^{2}\right) \frac{1}{d_{\alpha}(D)}
$$

for $|\alpha|=\alpha_{1}+\ldots+\alpha_{n}>1$. We do not present the proof of (16), because it repeats the proof for polydisk $U_{1}$ (see [4]), but deals with inequalities (15) instead of Cauchy inequalities. If (4) holds in $D$, then, applying (16), we get

$$
\begin{aligned}
\sum_{\alpha} \sup _{D_{r}}\left|c_{\alpha} z^{\alpha}\right| & =\sum_{\alpha}\left|c_{\alpha}\right| d_{\alpha}\left(D_{r}\right) \\
& \leq\left|c_{0}\right|+\left(1-\left|c_{0}\right|^{2}\right) \sum_{|\alpha|=1}^{\infty} \frac{d_{\alpha}\left(D_{r}\right)}{d_{\alpha}(D)} \\
& =\left|c_{0}\right|+\left(1-\left|c_{0}\right|^{2}\right) \sum_{k=1}^{\infty}\left(\begin{array}{c}
n+k-1 \\
k
\end{array}\right) r^{k} \\
& =\left|c_{0}\right|+\left(1-\left|c_{0}\right|^{2}\right)\left[\frac{1}{(1-r)^{n}}-1\right] .
\end{aligned}
$$

Now if

$$
\frac{1}{(1-r)^{n}}-1 \leq \frac{1}{2}
$$

then

$$
\sum_{\alpha} \sup _{D_{r}}\left|c_{\alpha} z^{\alpha}\right| \leq\left|c_{0}\right|+\left(1-\left|c_{0}\right|^{2}\right) \frac{1}{2}=1-\frac{1}{2}\left(1-\left|c_{0}\right|\right)^{2}<1 .
$$

The condition (17) means that (7) is true if $r \leq 1-\sqrt[n]{\frac{2}{3}}$.

Proof of Theorem 5. Consider Borel probability measure on $\partial D^{1}$, which is invariant under all unitary transformations of $\mathbf{C}^{n}$ :

$$
d \mu=\frac{(n-1) !}{(2 \pi i)^{n}} d\left|z_{1}\right|^{2} \wedge \cdots \wedge d\left|z_{n-1}\right|^{2} \wedge \frac{d z_{1}}{z_{1}} \wedge \cdots \wedge \frac{d z_{n}}{z_{n}} .
$$

The monomials $z^{\alpha}$ are orthogonal with respect to integration by $\mu$ and

$$
\int_{\partial D^{1}}\left|z^{2 \alpha}\right| d \mu=\frac{\alpha_{1} ! \cdots \alpha_{n} !(n-1) !}{(|\alpha|+n-1) !} .
$$


Next, we repeat the proof of Theorem 2 from [4] using Wiener's method, but integrating on the sphere $\partial D^{1}$ with respect to the measure $\mu$ instead of integrating on the unit torus as in [4]. Thus, we obtain

$$
\sum_{|\alpha|=k}\left|c_{\alpha}\right|^{2} \frac{\alpha_{1} ! \cdots \alpha_{n} !(n-1) !}{(|\alpha|+n-1) !} \leq\left(1-\left|c_{0}\right|^{2}\right)^{2}
$$

Furthermore, notice that from the Schwarz Lemma for the ball $D^{1}$ it follows (again by using Wiener's method from [4]) that

$$
\sum_{|\alpha|=1}\left|c_{\alpha}\right|^{2} \leq\left(1-\left|c_{0}\right|^{2}\right)^{2}
$$

Then, recalling that

$$
d_{\alpha}\left(D^{1}\right)=\sqrt{\frac{\alpha_{1}^{\alpha_{1}} \cdots \alpha_{n}^{\alpha_{n}}}{|\alpha|^{|\alpha|}}}
$$

where $0^{0}=1$, we get

$$
\begin{aligned}
& \sum_{\alpha} \sup _{D_{r}}\left|c_{\alpha} z^{\alpha}\right|=\left|c_{0}\right|+\left(\sum_{|\alpha|=1}\left|c_{\alpha}\right|\right) r+\sum_{k=2}^{\infty} \sum_{|\alpha|=k}\left|c_{\alpha}\right| \sqrt{\frac{\alpha_{1}^{\alpha_{1}} \cdots \alpha_{n}^{\alpha_{n}}}{|\alpha|^{|\alpha|}}} r^{k} \\
& \quad \leq\left|c_{0}\right|+\left(1-\left|c_{0}\right|^{2}\right) r \sqrt{n}+\left(1-\left|c_{0}\right|^{2}\right) \sum_{k=2}^{\infty}\left(\sum_{|\alpha|=k} \frac{(k+n-1) ! \alpha_{1}^{\alpha_{1}} \cdots \alpha_{n}^{\alpha_{n}}}{k^{k} \alpha_{1} ! \cdots \alpha_{n} !(n-1) !}\right)^{1 / 2} r^{k} \\
& \quad<\left|c_{0}\right|+\left(1-\left|c_{0}\right|^{2}\right) r \sqrt{n}+\left(1-\left|c_{0}\right|^{2}\right) \sum_{k=2}^{\infty}\left(\frac{(k+n-1) !}{k !(n-1) !} \sum_{|\alpha|=k} \frac{k !}{\alpha_{1} ! \cdots \alpha_{n} !}\right)^{1 / 2} r^{k} \\
& \quad=\left|c_{0}\right|+\left(1-\left|c_{0}\right|^{2}\right) r \sqrt{n}+\left(1-\left|c_{0}\right|^{2}\right) \sum_{k=2}^{\infty} \sqrt{\left(\begin{array}{c}
k+n-1 \\
k
\end{array}\right)}(r \sqrt{n})^{k} .
\end{aligned}
$$

It remains now to apply the estimate, obtained in Remark 1 from [4].

Proof of Theorem 6. Consider the function

$$
f_{a}(z)=\frac{1+a}{2} \frac{1-\left(z_{1}+\cdots+z_{n}\right)}{1-a\left(z_{1}+\cdots+z_{n}\right)}=\sum_{\alpha} c_{\alpha} z^{\alpha}
$$

where $0<a<1$. Then $\left|f_{a}(z)\right|<1$ in hypercone $D^{\circ}$ and

$$
\sum_{\alpha}\left|c_{\alpha} z^{\alpha}\right|=\frac{1+a}{2}+\frac{1-a^{2}}{2} \sum_{k=1}^{\infty} a^{k-1} \sum_{|\alpha|=k} \frac{k !}{\alpha_{1} ! \cdots \alpha_{n} !}\left|z^{\alpha}\right|
$$

Since

$$
d_{\alpha}\left(D^{\circ}\right)=\frac{\alpha_{1}^{\alpha_{1}} \cdots \alpha_{n}^{\alpha_{n}}}{|\alpha|^{|\alpha|}}
$$


it follows that

$$
\begin{aligned}
& \sum_{\alpha}\left|c_{\alpha}\right| d_{\alpha}\left(D_{r}^{\circ}\right)=\frac{1+a}{2}+\frac{1-a^{2}}{2} \sum_{k=1}^{\infty} \sum_{|\alpha|=k} a^{k-1} \frac{k ! \alpha_{1}^{\alpha_{1}} \cdots \alpha_{n}^{\alpha_{n}}}{\alpha_{1} ! \cdots \alpha_{n} ! k^{k}} r^{k} \\
> & \frac{1+a}{2}+\frac{1-a^{2}}{2} \sum_{k=1}^{\infty} a^{k-1} \frac{k !}{k^{k}}\left(\sum_{|\alpha|=k} \frac{1}{\alpha_{1} ! \cdots \alpha_{n} !}\right) r^{k} \\
= & \frac{1+a}{2}+\frac{1-a^{2}}{2 a} \sum_{k=1}^{\infty} \frac{(a n r)^{k}}{k^{k}} \geq 1,
\end{aligned}
$$

if

$$
\sum_{k=1}^{\infty} \frac{(a n r)^{k}}{k^{k}} \geq \frac{a}{1+a}
$$

Let $x_{0}(a)$ be the root of the equation

$$
\sum_{k=1}^{\infty} \frac{x^{k}}{k^{k}}=\frac{a}{1+a}
$$

then, if anr $\geq x_{0}(a)$, (7) fails for that $r$ and $D=D^{\circ}$. Now, considering $a \rightarrow 1$, we obtain that $(7)$ is not true for $D=D_{\text {。 }}$ if $r \geq \frac{x_{0}}{n}$, where $x_{0}=x_{0}(1)$. Notice that $x_{0}$ is a root of the equation

$$
\sum_{k=1}^{\infty} \frac{x^{k}}{k^{k}}=1 / 2
$$

hence $B_{n}\left(D^{\circ}\right) \leq x_{0} / n$. Using the program "Mathematica 3.0" [7], we estimated $x_{0}$ from above. We obtained that the equation

$$
\sum_{k=1}^{p} \frac{x^{k}}{k^{k}}=1 / 2
$$

has a root 0.446662 (where the last decimal digit is precise) if $p$ runs from 5 till 25 . So, the equation (20) has a root less than 0.446663 if $5 \leq p \leq 25$; hence this estimate is true for $x_{0}$.

Proof of Theorem \%. Notice that by analogy with (18) it is true that

$$
\int_{\partial D_{r}^{\circ}}\left|z^{\alpha}\right| d \mu_{r}=\frac{\alpha_{1} ! \cdots \alpha_{n} !(n-1) !}{(|\alpha|+n-1) !} r^{|\alpha|} .
$$

Using (16), we get

$$
\begin{aligned}
& \sum_{\alpha}\left\|c_{\alpha} z^{\alpha}\right\|_{L^{1}\left(\partial D_{r}^{\circ}\right)} \\
& \quad \leq\left|c_{0}\right|+\left(1-\left|c_{0}\right|^{2}\right) \sum_{k=1}^{\infty} \sum_{|\alpha|=k} \frac{k^{k}(n-1) !}{(n+k-1) !} \frac{\alpha_{1} ! \cdots \alpha_{n} !}{\alpha_{1}^{\alpha_{1}} \cdots \alpha_{n}^{\alpha_{n}}} r^{|\alpha|} \\
& \quad<\left|c_{0}\right|+\left(1-\left|c_{0}\right|^{2}\right) \sum_{k=1}^{\infty} \frac{k^{k}(n-1) !}{(n+k-1) !} \sum_{|\alpha|=k} r^{|\alpha|} \\
& \quad=\left|c_{0}\right|+\left(1-\left|c_{0}\right|^{2}\right) \sum_{k=1}^{\infty} \frac{k^{k}}{k !} r^{k}
\end{aligned}
$$


Denote by $r_{0}$ the root of the equation

$$
\sum_{k=1}^{\infty} \frac{k^{k}}{k !} r^{k}=\frac{1}{2}
$$

Then (11) holds for $r=r_{0}$; therefore $L_{n}\left(D^{\circ}\right) \geq r_{0}$. The identity

$$
\sum_{k=1}^{\infty} \frac{k^{k}}{k !} x^{k} e^{-k x}=-1+\frac{1}{1-x}
$$

holds for $x$ in a neighborhood of 0 . This can be verified by computing the Maclaurin series coefficients of the left-hand side and observing that they reduce to the value 1 by a standard theorem on sums of binomial coefficients. Now put $x=1 / 3$ in equation (22) to recognize the solution of equation (21).

Finally, consider, as in the proof of Theorem 6 , the function $f_{a}(z)$ for which

$$
\begin{aligned}
\sum_{\alpha}\left\|c_{\alpha} z^{\alpha}\right\|_{L^{1}\left(\partial D_{r}^{\circ}\right)} & =\frac{1+a}{2}+\frac{1-a^{2}}{2} \sum_{k=1}^{\infty} \frac{k !(n-1) ! a^{k-1}}{(n+k-1) !} \sum_{|\alpha|=k} r^{|\alpha|} \\
& =\frac{1+a}{2}+\frac{1-a^{2}}{2 a} \sum_{k=1}^{\infty}(a r)^{k} \geq 1,
\end{aligned}
$$

if

$$
\sum_{k=1}^{\infty}(a r)^{k} \geq \frac{a}{1+a}
$$

When $a \rightarrow 1$, we get that the inequality (11) fails if $r>1 / 3$.

Proof of Theorem 8. In each section of the domain $Q$ by a complex line

$$
\alpha=\left\{z: z_{j}=a_{j} t, \quad j=1, \cdots, n ; \quad t \in \mathbf{C}\right\}
$$

the series turns into the power series by $t$

$$
f(a t)=\sum_{k=0}^{\infty} P_{k}(a) t^{k}
$$

and, in addition, $|f(a t)|<1$. By Theorem 1

$$
\sum_{k=0}^{\infty}\left|P_{k}(a) t^{k}\right|<1
$$

in the section $\alpha \cap\left(\frac{1}{3} \cdot Q\right)$. But it is just (14), since $\alpha$ is an arbitrary complex line passing through the origin. Conversely, let the domain $Q$ be convex; then $Q$ is an intersection of half-spaces

$$
Q=\bigcap_{a \in J}\left\{z: \operatorname{Re}\left(a_{1} z_{1}+\cdots+a_{n} z_{n}\right)<1\right\}
$$

for some $J$. Since $Q$ is circular, we obtain

$$
Q=\bigcap_{a \in J}\left\{z:\left|a_{1} z_{1}+\cdots+a_{n} z_{n}\right|<1\right\} .
$$

It is sufficient now to show that the constant $1 / 3$ cannot be improved for each domain $P_{a}=\left\{z:\left|a_{1} z_{1}+\ldots+a_{n} z_{n}\right|<1\right\}$. From Theorem 1 it follows that for any $r>1 / 3$ there exists a function $f(z)$, represented by (1) and such that $|f(z)|<1$ in 
the unit disk, but (2) fails in the disk $\{z:|z|<r\}$. To complete the proof we use the functions $f\left(a_{1} z_{1}+\ldots+a_{n} z_{n}\right)$.

Proof of Theorem 9. If $z \in r \cdot D^{\circ}$, then from (16) it follows

$$
\begin{gathered}
\sum_{\alpha}\left|c_{\alpha} z^{\alpha}\right| \leq\left|c_{0}\right|+\left(1-\left|c_{0}\right|^{2}\right) \sum_{|\alpha|=1}^{\infty} \frac{|\alpha|^{|\alpha|}}{\alpha_{1}^{\alpha_{1}} \ldots \alpha_{n}{ }^{\alpha_{n}}}\left|z^{\alpha}\right| \\
=\left|c_{0}\right|+\left(1-\left|c_{0}\right|^{2}\right) \sum_{|\alpha|=1}^{\infty} \frac{|\alpha|^{|\alpha|}}{|\alpha| !} \frac{\alpha_{1} ! \ldots \alpha_{n} !}{\alpha_{1}^{\alpha_{1}} \ldots \alpha_{n}{ }^{\alpha_{n}}} \frac{|\alpha| !}{\alpha_{1} ! \ldots \alpha_{n} !}\left|z^{\alpha}\right| \\
<\left|c_{0}\right|+\left(1-\left|c_{0}\right|^{2}\right) \sum_{k=1}^{\infty} \frac{k^{k}}{k !} r^{k} .
\end{gathered}
$$

Thus (5) holds for $r>r_{0}$, where $r_{0}$ is the root of equation (21).

Suppose $z \notin 1 / 3 \cdot D^{\circ}$; then we have for the function $f_{a}(z)$ (which was used in the proof of Theorem 6 )

$$
\sum_{\alpha}\left|c_{\alpha} z^{\alpha}\right|=\frac{1+a}{2}+\frac{1-a^{2}}{2} \sum_{k=1}^{\infty} a^{k-1}\left(\left|z_{1}\right|+\ldots+\left|z_{n}\right|\right)^{k} \geq 1
$$

if

$$
\sum_{k=1}^{\infty} a^{k}\left(\left|z_{1}\right|+\ldots+\left|z_{n}\right|\right)^{k} \geq \frac{a}{1+a} .
$$

Hence inequality (5) fails as $a \rightarrow 1$.

\section{Final Remarks}

Remark 2. In the proof of Theorem 5 we used the facts that in equality (18) the domain in consideration is the ball $D^{1}$ and that the estimates $d_{\alpha}\left(D^{1}\right) \leq 1$ are valid. Therefore, an analogous theorem holds for any complete $n$-circular domain $D$ if $D^{1} \subset D \subset U_{1}$. But for the hypercone $D^{\circ}$ this is not true (see Theorem 6).

Remark 3. In the proof of Theorem 6 was used the following quite rough inequality:

$$
\sum_{|\alpha|=k} \frac{\alpha_{1}^{\alpha_{1}} \cdots \alpha_{n}^{\alpha_{n}}}{\alpha_{1} ! \cdots \alpha_{n} !} \geq \sum_{|\alpha|=k} \frac{1}{\alpha_{1} ! \cdots \alpha_{u} !} .
$$

Therefore, in fact, the estimate from above in the theorem might be decreased. For example, if $n=2(10)$ implies $B_{2}\left(D^{\circ}\right)<0.223332$, but using a PC one can show that $B_{2}\left(D^{\circ}\right)<0.191373$. Hence, $0.183502<B_{2}\left(D^{\circ}\right)<0.191373$.

Remark 4 . Comparing Theorem 2 and the corollary we can see that $B_{n}(D)$ depends essentially on the domain $D$ since $B_{n}\left(U_{1}\right)=K_{n}$. It seems that it is more natural to consider not a single number in the problem considered in Theorem 2 and Theorem 3 , but the largest subdomain $D_{B}$ of $D$ such that (5) holds. From [4] it follows, for example, that $U_{1 B}$ contains the ball $\frac{1}{3} \cdot D^{1}$, and from Theorem 9 it follows that $D^{\circ}{ }_{B} \subset 1 / 3 \cdot D^{\circ}$.

Remark 5. Notice that there exist such unbounded $n$-circular domains $D$, for which the problems defined by the conditions (5) and (7) are equivalent. Consider, for example, the domains $D=\left\{z:|z|^{\beta}<c\right\}$, where $\beta$ is a multiindex with coprime components. Bounded holomorphic functions in such domains depend only on one 
variable $z^{\beta}$ and the exact value of Bohr's radius equals $\left(\frac{1}{3}\right)^{1 /|\beta|}$. Thus there exist $n$ circular domains with Bohr's radius arbitrarily close to 1 . Therefore it is impossible to remove the assumption about convexity in Theorem 8 .

Remark 6. Unlike Theorems 1-7, in Theorem 8 the series (13) is not a basis expansion. In [1] it was shown that there exists a basis in the space of all holomorphic functions in $Q$, consisting of homogeneous polynomials $P_{k, m}(z)$, where $k$ is the degree of the polynomial and $m=1, \ldots,\left(\begin{array}{c}k+n-1 \\ k\end{array}\right)$. It is reasonable to consider Bohr's problem for such basis expansions, but there are no results yet in this direction. This question is a particular case of a more general problem in Bohr spirit for expansions by an arbitrary basis in a domain $D \subset \mathbf{C}^{n}$ (under some restrictions on the basis, because, for example, the basis $\left\{1,(z-1) / 2, z^{2}, z^{3}, \ldots\right\}$ in the unit disk has no Bohr's constant).

\section{ACKNOWLEDGEMENT}

I am sincerely thankful to D. Khavinson, who turned my attention to Bohr's result (Theorem 1) and its first multidimensional analogues (Theorems 2 and 3), and to H. P. Boas, who suggested to me the exact solution of the equation (21) instead of the approximate one. I am also sincerely thankful to V. Zahariuta, P. Djakov and A. Vidras for their help during the preparation of the English version of the paper.

The results of this paper were obtained during the author's visit to the Middle East Technical University. The author would like to thank A. Aytuna for the invitation, and as well, A.Aytuna and B.Karasözen for their support.

\section{REFERENCES}

[1] L.A. Aizenberg, The spaces of functions analytic in $(p, q)$-circular regions, Soviet Math. Dokl. 2 (1960), 79-82. MR 26:359

[2] L.A. Aizenberg, B.S. Mityagin, The spaces of functions analytic in multicircular domains, Sibirsk. Math. Zh. 1 (1960), 153-170 (Russian). MR 23:A1838

[3] L.A. Aizenberg, A.P. Yuzhakov, Integral Representations and Residues in Multidimensional Complex Analysis, AMS, 1983, 283 pp. MR 85a:32006

[4] H.P. Boas, D. Khavinson, Bohr's power series theorem in several variables, Proc. Amer. Math. Soc. 125 (1997), 2975-2979. MR 98i:32002

[5] H. Bohr, A theorem concerning power series, Proc. London Math. Soc. (2) 13 (1914) 1-5.

[6] W.Rudin, Function theory in the unit ball of $\mathbf{C}^{n}$, Springer-Verlag, 1980, 436 pp. MR 82i:32002

[7] Wolfram Research, Mathematica 3.0, 1996.

Department of Mathematics, and Computer Science, Bar-Ilan University, 52900 RAMAT-GAN, ISRAEL

E-mail address: aizenbrg@macs.biu.ac.il 